\title{
Un caso di encefalopatia posteriore reversibile (PRES), sindrome neurologica acuta da edema cerebrale reversibile su base multifattoriale
}

\section{Posterior reversible encephalopathy syndrome (PRES), an acute neurological syndrome due to reversible multifactorial brain edema: a case report}

\author{
Camilla Cicognani ${ }^{a, *}$, Chiara Vezzadini ${ }^{a}$, Stella Battaglia ${ }^{b}$, \\ Anna F. Marliani ${ }^{\text {b }}$, Roberto Zoni ${ }^{a}$
}

\author{
a Divisione di Medicina Generale (Direttore: dott. Roberto Zoni), Ospedale "Bellaria", Azienda USL Città di Bologna \\ bervizio di Neuroradiologia, Ospedale "Bellaria", Azienda USL Città di Bologna
}

Ricevuto il 4 agosto 2009; accettato il 7 novembre 2009

disponibile online il 11 febbraio 2010

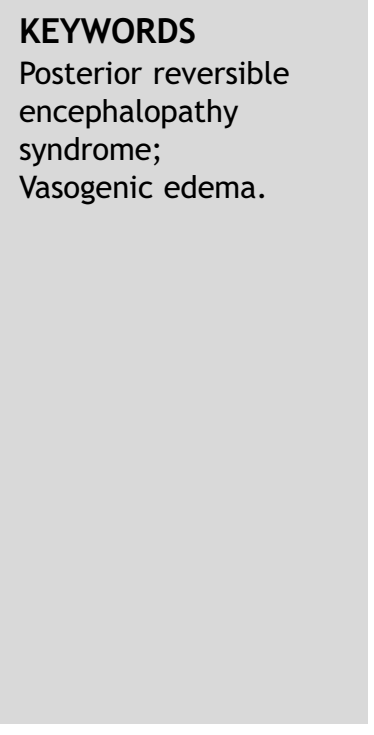

\begin{abstract}
Summary
Background: The essential features of Posterior Reversible Encephalopathy Syndrome (PRES) are headache, mental changes, seizures, visual symptoms and often arterial hypertension. Brain RMN typically shows cortico-sottocortical parieto-occipital edema, with a bilateral and symmetric distribution. PRES develops in clinical conditions as hypertensive encephalopathy, preeclampsia/ eclampsia, autoimmune diseases, after transplantation, infections and as an adverse effect of immunosuppressive drugs or chemotherapy. It usually completely reverses with treatment, although permanent sequelae are possible in case of delayed or missed diagnosis.

Case report: We describe the case of a transsexual $(M \rightarrow F)$ and tetraplegic patient, admitted for neck and low back pain. She suddenly developed headache, confusion, seizures and severe hypertension with normal blood tests. RMN showed multiple cortico-sottocortical areas of vasogenic and citotoxic edema in temporo-occipital, parietal, frontal, and cerebellar regions. Soon after the beginning of the antihypertensive therapy, clinical recovery was observed, as well as the disappearance of edema at RMN.

Discussion and conclusions: Although PRES is usually associated with definite pathological conditions, it is not always the case, as was for the patient here described, who had no predisposing factors in her past clinical history, and presented hypertension only in the acute phase of the syndrome. Since, moreover, PRES usually presents with acute non specific features and it can be misdiagnosed with other serious diseases, the clinician will be helped by the
\end{abstract}

\footnotetext{
* Corrispondenza: Divisione di Medicina Generale, Ospedale "Bellaria", via Altura 3 - 40139 Bologna.

E-mail: camilla.cicognani@ausl.bologna.it (C. Cicognani).
} 
knowledge of this syndrome to promptly start diagnostic workup and treatments, and avoid permanent neurological deficits.

(c) 2010 Elsevier Srl. All rights reserved.

\section{Introduzione}

La sindrome da encefalopatia posteriore reversibile (Posterior Reversible Encephalopathy Syndrome, PRES) è un'entità clinica a esordio acuto caratterizzata da cefalea, disturbo della coscienza, crisi convulsive e disturbi focali prevalentemente visivi, spesso associata a stato ipertensivo.

Diagnostico è il quadro RMN che mostra aree di edema vasogenico cortico-sottocorticali bilaterali e simmetriche, tipicamente in sede occipitale, parietale e cerebellare con emorragia petecchiale. Possibile è l'interessamento delle regioni temporali, frontali, dei gangli della base, del tronco encefalico e della sostanza bianca profonda. La presenza di aree di infarto e di emorragia si associa a una prognosi peggiore.

La PRES si sviluppa in condizioni cliniche specifiche, eterogenee tra di loro ma di frequente riscontro nei reparti internistici, quali stato ipertensivo, preeclampsia/eclampsia, malattie autoimmuni, dopo trapianto di midollo osseo e di organi solidi, stati infettivi/sepsi, terapie farmacologiche specifiche, in particolare con immunosoppressori [1].

L'eziopatogenesi non è tuttora definita. Le due principali ipotesi formulate (edema da iperperfusione vs edema da ipoperfusione) si basano su studi clinici e angiografici con risultati finora non concordi.

L'iperperfusione sarebbe conseguenza della perdita dell'autoregolazione vasculocerebrale in corso di encefalopatia ipertensiva, con prevalente interessamento delle regioni posteriori per la scarsa innervazione simpatica perivascolare del sistema vertebro-basilare [2].

In alternativa, l'ipoperfusione sarebbe causata da vasospasmo cerebrale per disfunzione endoteliale indotta dalla produzione di citochine. L'attivazione immune e infiammatoria sistemica sarebbe un processo patologico comune alle condizioni a rischio di PRES, comprese quelle non necessariamente associate a ipertensione arteriosa (20-30\% dei casi) [3,4].

L'evoluzione clinica della PRES è di solito favorevole. Il controllo pressorio e il trattamento delle condizioni associate

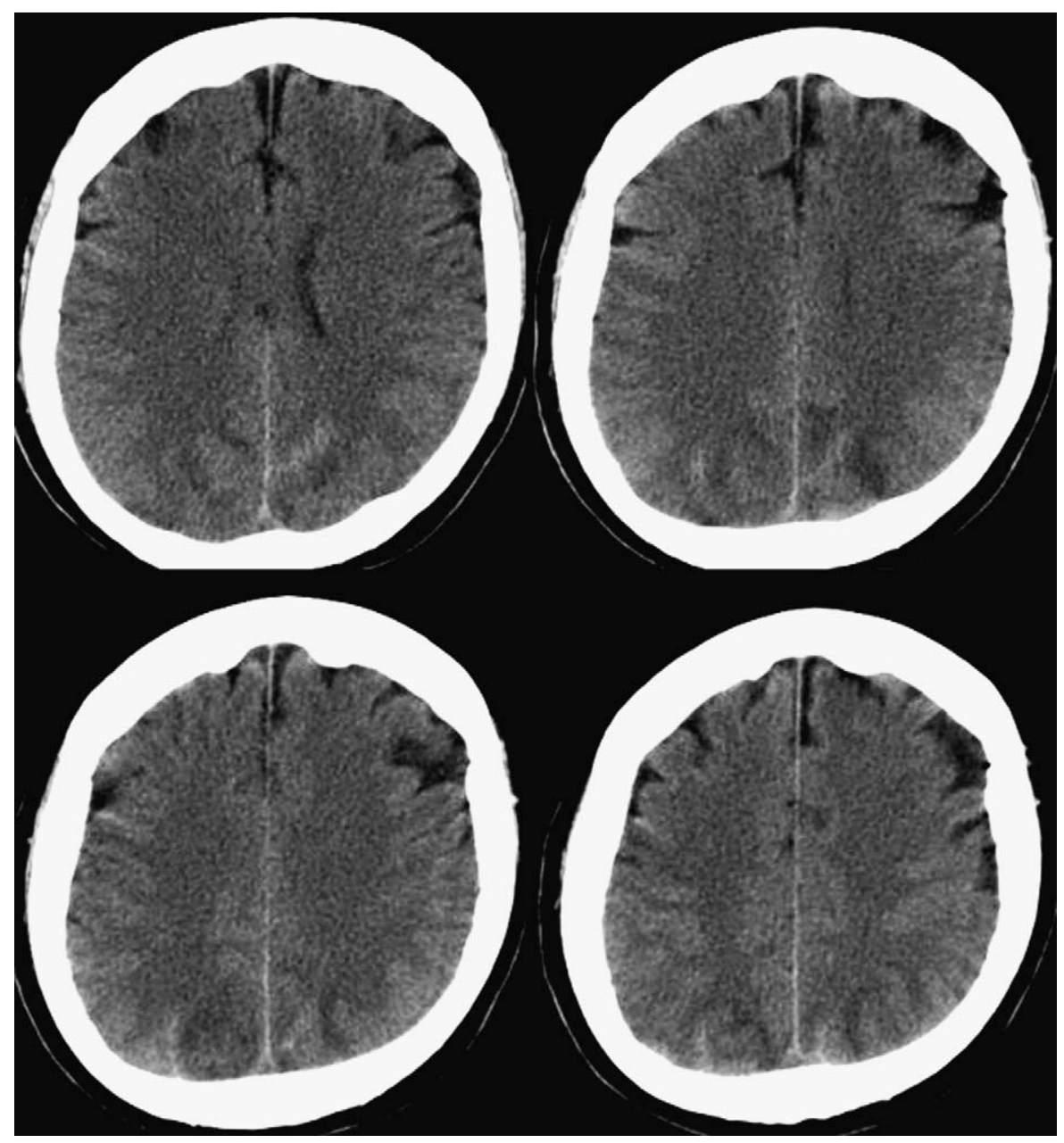

Figura 1 TC senza/con mezzo di contrasto: sfumate aree ipodense cortico-sottocorticali occipito-parietali bilaterali e simmetriche. 
producono una regressione delle alterazioni cliniche e radiologiche nella quasi totalità dei casi. Tuttavia, in presenza di un quadro neuroradiologico sfavorevole o nel caso di mancato riconoscimento della sindrome con ritardo terapeutico, può risultare un danno neurologico permanente, spesso esito di secondario infarto o emorragia cerebrale.

\section{Caso clinico}

Descriviamo il caso di una paziente transessuale $(\mathrm{M} \rightarrow \mathrm{F})$ di 46 anni, con tetraplegia post-traumatica e sindrome ansiosodepressiva, ricoverata per una riacutizzazione di dolore al rachide cervicale e lombosacrale.

Per la stessa problematica, la paziente era stata sottoposta nei mesi precedenti a una RMN del rachide che aveva evidenziato esiti di mielopatia cervicale C5-C6, discopatia L4-L5 e L5-S1, in assenza di ernie, stenosi del canale vertebrale o alterazioni del cono midollare.

A domicilio la paziente assumeva carbamazepina, baclofene, sertralina, quetiapina, tramadolo e, al momento del ricovero, non stava effettuando una terapia estrogenica sostitutiva.

In prima giornata di ricovero la paziente presenta intensa rachialgia, nausea e ipertensione sistolica di grado lieve $(150 / 80 \mathrm{mmHg})$. Non vi sono febbre e segni meningei né alterazioni dello stato di coscienza.

Il quadro clinico cambia improvvisamente in seconda giornata con la comparsa di agitazione psicomotoria, confusione ed eloquio incoerente. I valori pressori sono normali.

L'EEG non mostra anomalie epilettiformi.

La TC dell'encefalo senza/con mezzo di contrasto mostra sfumate aree ipodense cortico-sottocorticali in regione occipito-parietale bilateralmente con strie iperdense, sospette per lesioni ischemiche con minimo infarcimento ematico. La somministrazione di mezzo di contrasto non modifica il quadro (fig. 1).

L'angioTC cerebrale e dei vasi del collo non mostra occlusioni arteriose, trombosi venose o dissezioni vascolari.

Segue una crisi epilettica generalizzata.

In terza giornata compare ipertensione arteriosa severa $(190 / 130 \mathrm{mmHg})$.

In anestesia generale viene eseguita una RMN senza/con mezzo di contrasto, con studio di diffusione, che mostra multiple aree di alterato segnale, cortico-sottocorticali, confluenti fra loro, disomogeneamente iperintense nelle sequenze $\mathrm{T} 2$ pesate, e ipointense in $\mathrm{T} 1$, in sede occipitale, parietale, temporale e cerebellare bilateralmente. Analoghi focolai, più piccoli, sono presenti anche in sede frontale posteriore e corona radiata da ambo i lati, in sede talamocapsulare a destra e, infine, nella capsula esterna di sinistra; si associano segni di microemorragia. Lo studio di diffusione, completato con il calcolo della mappa del coefficiente di diffusione apparente (ADC), conferma, nelle suddette aree di alterato segnale, la presenza di zone a elevata diffusione e di altre a diffusione ristretta, in rapporto alla contemporanea presenza di edema vasogenico e citotossico.

Nel complesso, i reperti sono compatibili con un quadro di PRES (figg. 2 e 3).

La valutazione anamnestica, laboratoristica e strumentale non identifica altre condizioni associate, a parte lo stato ipertensivo.

Le indagini ormonali per ipertensione secondaria evidenziano ipercortisoluria (cortisolo urinario: $600 \mathrm{microg} / \mathrm{die}, \mathrm{vn}$ : 10-100), cortisolo sierico e ACTH nella norma. Il test di soppressione con desametazone $1 \mathrm{mg}$ mostra una normale

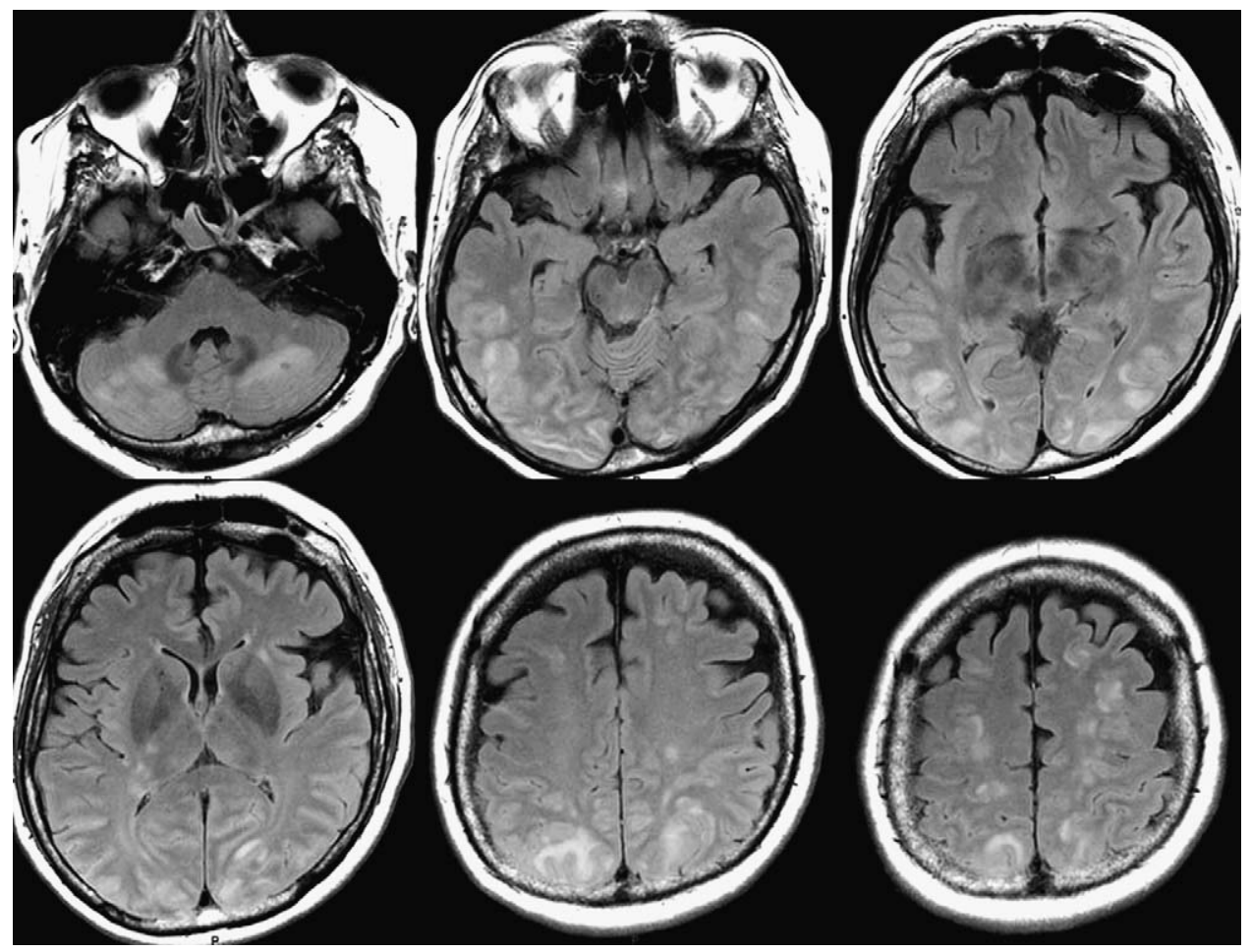

Figura 2 RMN, sequenze assiali FLAIR T2 pesate: multiple aree iperintense, cortico-sottocorticali, confluenti fra loro, ai lobi temporali, occipitali, fronto-parietali e cerebellari da ambo i lati. 


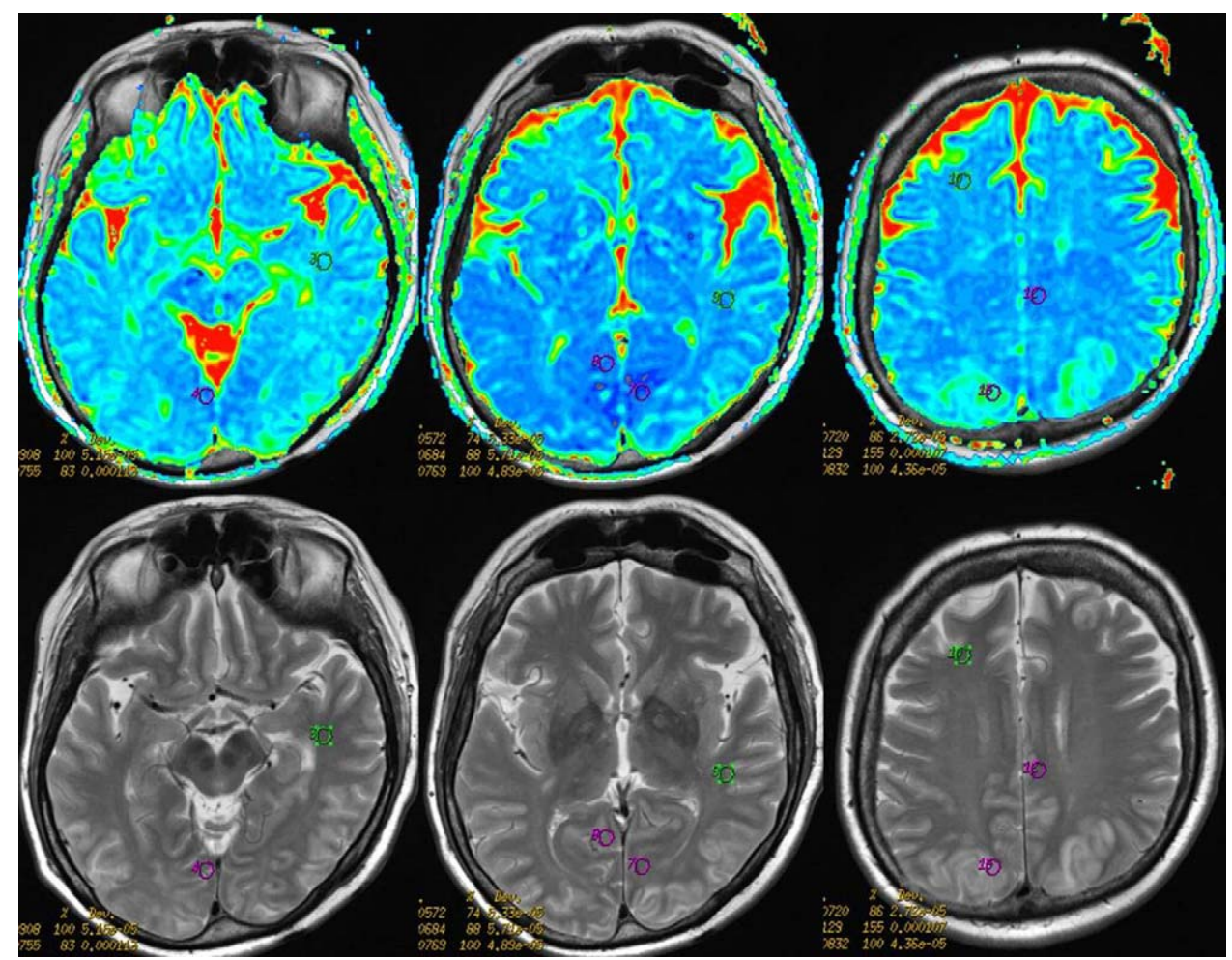

Figura 3 Mappa parametrica del coefficiente di diffusione apparente (ADC): sono state posizionate numerose aree di interesse (ROI) nelle zone di alterato segnale, evidenziate nella sequenza di diffusione, corrispondenti a quelle visibili nella sequenza T2 pesata. I valori di ADC risultano in alcune zone ridotti, espressione di edema citotossico (nella mappa di ADC sono rappresentate in blu) e in altre zone aumentati, espressione di edema vasogenico (nella mappa di ADC sono rappresentate in verde).

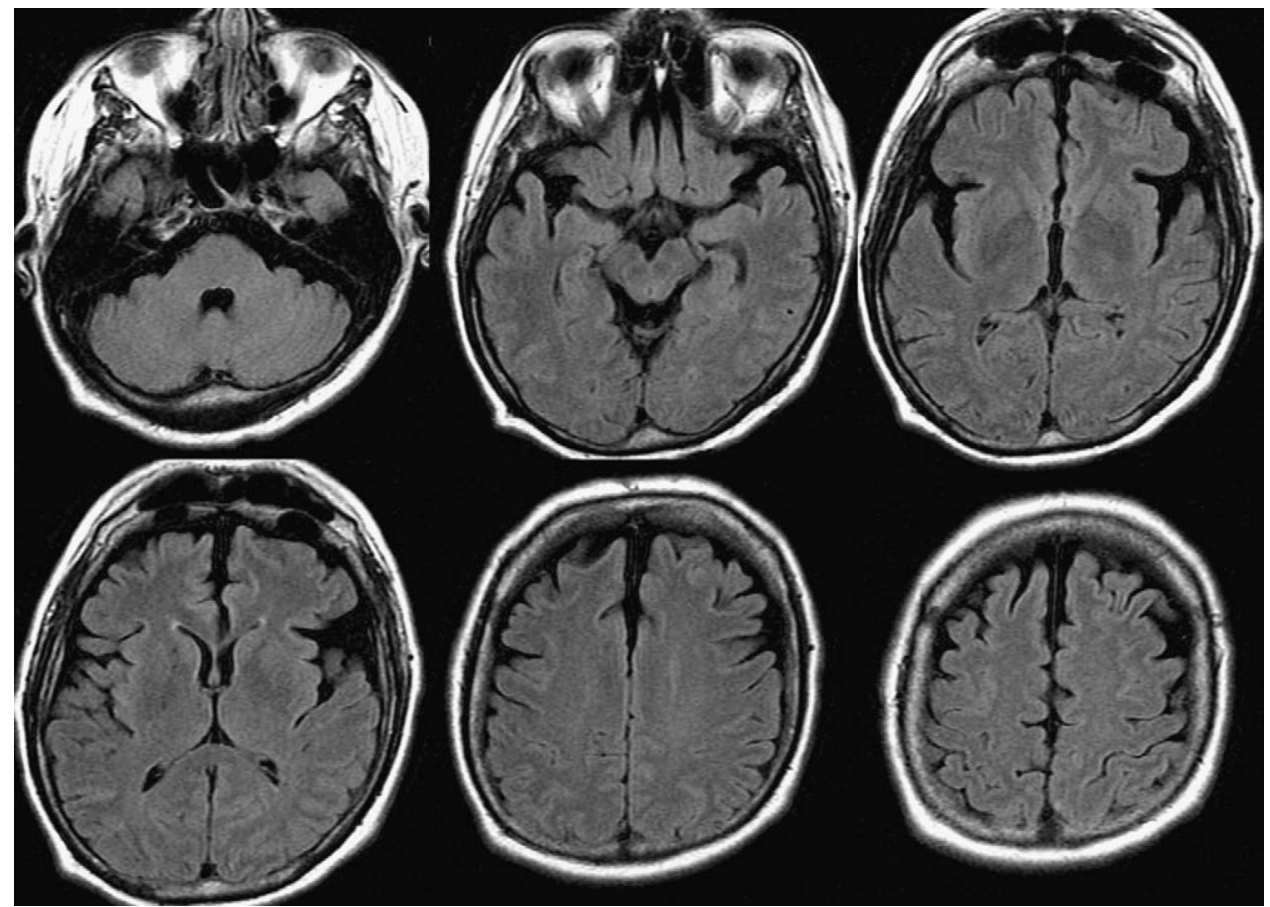

Figura 4 Controllo RMN a un mese, sequenze assiali FLAIR T2 pesate: regressione neuroradiologica. 
risposta. Nella norma sono: metanefrine urinarie, aldosterone, attività reninina plasmatica.

Viene iniziato un trattamento con calcio antagonisti (amlodipina $5 \mathrm{mg} / \mathrm{die}$ ) con rapida regressione del disturbo di coscienza e più graduale controllo dell'ipertensione.

Il controllo RMN a un mese mostra una sostanziale normalizzazione neuroradiologica ( fig. 4).

Una rivalutazione a 6 mesi conferma la normalità dei reperti ormonali e neuroradiologici.

Gli Autori dichiarano che lo studio presentato è stato realizzato in accordo con gli standard etici stabiliti nella Dichiarazione di Helsinki, e che il consenso informato è stato ottenuto da tutti i partecipanti prima del loro arruolamento allo studio.

\section{Discussione}

La PRES è un'entità clinica rara. Secondo una revisione della letteratura anglosassone dal 1996 al 2007, i casi descritti sono 170 , con una maggiore incidenza negli ultimi anni in rapporto a una cresciuta attenzione clinica e alla disponibilità della RMN. Rispetto alla TC, la RMN presenta, infatti, sensibilità e specificità superiori, in particolare grazie agli studi funzionali, come la diffusione, per la caratterizzazione delle aree di edema vasogenico/citotossico [5].

Vi è poi una frequenza specifica di PRES nelle diverse condizioni patologiche. In particolare, dei 170 casi sopra menzionati 17 presentavano un lupus prediagnosticato. Altre revisioni mostrano stime non dissimili di frequenza di PRES in corso di lupus eritematoso sistemico (12-46 casi totali fino agli anni 2005-2008).

La PRES si presenta dopo trapianto d'organo solido nello 0,4-6\% dei casi e fino al 7-9\% dopo trapianto di midollo osseo allogenico [1].

La conoscenza della PRES è di interesse per l'internista per l'eterogeneità delle condizioni predisponenti.

Si riconoscono patologie tipicamente a rischio, farmaci predisponenti e patologie occasionalmente associate (tabelle 1 e 2).

Nell'ambito delle malattie autoimmuni, la differenziazione tra PRES, lupus neuropsichiatrico e altre complicanze del lupus eritematoso sistemico a carico del sistema nervoso centrale può presentare difficoltà a causa della frequente sovrapposizione delle manifestazioni cliniche.

Nel lupus eritematoso sistemico la PRES non è una manifestazione primitiva della malattia, ma una complicanza secondaria a diversi fattori, quali lo stato ipertensivo associato a riacutizzazione di glomerulonefrite, il recente inizio o

Tabella 1 Condizioni patologiche tipiche a rischio di PRES.

Encefalopatia ipertensiva

Preeclampsia/eclampsia

Malattie autoimmuni (lupus eritematoso sistemico, sindrome di Wegener, sclerosi sistemica, panarterite nodosa)

Post-trapianto (midollo osseo allogenico/organo solido)

Stati infettivi/sepsi

Farmaci
Tabella 2 Farmaci predisponenti alla PRES e condizioni occasionalmente associate.

Farmaci

- Ciclosporina

- Tacrolimus

- Citarabina

- Cisplatino

- Gemcitabina

- Bevacizumab

- Immunoglobuline ev

- Eritropoietina

- Terapia steroidea ad alte dosi

Patologie

- Feocromocitoma

- Porfiria acuta intermittente

- Porpora trombotica trombocitopenica

- Ipercalcemia

- Sindrome di Guillain-Barré

l'aumento di dosaggio di farmaci immunosoppressori, la terapia con metilprednisolone ad alte dosi.

In presenza di questi aspetti e della sintomatologia tipica va posto il sospetto clinico di PRES. In caso di conferma neuroradiologica va mantenuta la terapia immunosoppressiva, se la malattia è attiva, e vanno trattate le manifestazioni cliniche della sindrome $[5,6]$.

Un altro aspetto da considerare è il rapporto tra PRES ed encefalopatia ipertensiva.

La definizione "encefalopatia ipertensiva" fu coniata nel 1928 da Oppenheimer e Fishberg per descrivere una sindrome neurologica associata a ipertensione maligna. Grazie al migliore trattamento dell'ipertensione arteriosa, oggi i casi di ipertensione maligna sono molto rari e la sindrome si riscontra in pazienti normotesi che presentano acute elevazioni dei valori pressori.

Le manifestazioni cliniche dell'encefalopatia ipertensiva corrispondono a quelle della PRES: cefalea, segni neurologici focali, spesso disturbi visivi, convulsioni, stato di coscienza alterato, coma.

Le indagini neuroradiologiche mostrano tipicamente un edema (citotossico e vasogenico) cortico-sottocorticale che interessa principalmente i lobi occipitali e parietali, ma può coinvolgere i lobi temporali, frontali, i nuclei della base, il tronco encefalico e gli emisferi cerebellari. Le alterazioni del tessuto encefalico, di norma, regrediscono con il controllo dei valori pressori, ma in alcuni casi possono evolvere in lesioni ischemiche e/o emorragiche con esiti permanenti [2].

Tabella 3 PRES: diagnosi differenziale.

Trombosi venosa cerebrale

Infarto ischemico

Vasculite

Encefalite infettiva

Leucoencefalopatia multifocale progressiva

Encefalomielite acuta disseminata

Gliomatosi cerebrale 
L'encefalopatia ipertensiva si presenta pertanto con il quadro clinico e neuroradiologico della PRES, il quale, tuttavia, può realizzarsi anche in assenza di ipertensione arteriosa (20-30\% dei casi).

La PRES, a causa della presentazione acuta e aspecifica, deve essere posta in diagnosi differenziale con altre patologie rilevanti del sistema nervoso centrale (tabella 3).

Tra le malattie cerebrovascolari, la trombosi venosa cerebrale più di altre può presentarsi con i caratteri clinici e radiologici della PRES. L'esordio è acuto o, più spesso, subacuto. Le manifestazioni cliniche tipiche sono cefalea, convulsioni, alterazioni visive, deficit focali, disturbo della coscienza fino al coma e papilledema, in varia associazione tra di loro [7].

Tra i reperti neuroradiologici che contraddistinguono la trombosi dei seni venosi vi sono anormalità tissutali quali edema ed emorragia; lo studio angioTC venoso permette una diagnosi nella quasi totalità dei casi [8].

La paziente da noi descritta non aveva una storia clinica di ipertensione arteriosa e presentava valori elevati di cortisolo libero urinario. Ulteriori test diagnostici escludevano una sindrome di Cushing e mostravano una normale funzione cortico-surrenalica.

È ipotizzabile che dolore, stress, ospedalizzazione e la terapia con carbamazepina abbiano indotto in questa paziente una temporanea condizione di pseudosindrome di Cushing e il riscontro di una elevata cortisoluria [9].

Nella letteratura è descritto un caso di PRES favorita dallo stato ipertensivo in una bambina affetta da sindrome di Cushing. Sono riportati casi insorti in corso di terapia steroidea ad alte dosi.

È quindi possibile, analogamente, che lo sviluppo dello stato ipertensivo e della PRES nella nostra paziente sia risultato dall'effetto combinato di una temporanea iperfunzione cortico-surrenalica, del dolore e dello stress. Non si può escludere, inoltre, un'influenza specifica del dolore cervicale sull'emodinamica cerebrale [10].

Infine, occorre considerare se il particolare assetto ormonale della nostra paziente possa avere contribuito allo sviluppo di PRES. A causa del pregresso intervento chirurgico per transessualismo e dell'assenza di una terapia estrogenica sostitutiva al momento del ricovero, la paziente si trovava in uno stato di ipogonadismo, dato confermato dalle indagini di laboratorio. È nostra opinione che le alterazioni degli ormoni sessuali non abbiano avuto un ruolo determinante nello sviluppo della sindrome. Non vi sono, a nostra conoscenza, dati di letteratura indicativi di una predisposizione diretta alla PRES in presenza di alterazioni degli ormoni sessuali né, più in specifico, di casi di PRES in corso di transessualismo [11].

\section{Conclusioni}

La PRES è una sindrome neurologica acuta con manifestazioni non specifiche e pone, pertanto, problemi di diagnostica differenziale con altre gravi patologie del sistema nervoso centrale. Il tempestivo sospetto clinico consente, in genere, di iniziare l'iter diagnostico e i trattamenti necessari al fine di evitare danni neurologici permanenti. La conoscenza della sindrome e delle condizioni patologiche associate costituisce, quindi, un utile strumento per l'orientamento clinico.

È tuttavia possibile, come nel caso descritto, non ritrovare con certezza elementi predisponenti, nonostante un estensivo work-up diagnostico. Ne consegue l'utilità di ulteriori studi sui meccanismi fisiopatologici della PRES e i loro rapporti con le condizioni associate a tale patologia.

\section{Conflitto di interesse}

Gli autori dichiarano di essere esenti da conflitto d'interesse.

\section{Bibliografia}

[1] Bartynski WS. Posterior reversible encephalopathy syndrome, part 1: fundamental imaging and clinical features. AJNR Am J Neuroradiol 2008;29(6):1036-42.

[2] Schwartz RB. Hyperperfusion encephalopathies: hypertensive encephalopathy and related conditions. Neurologist 2002;8(1): 22-34.

[3] Bartynski WS. Posterior reversible encephalopathy syndrome, part 2: controversies surrounding pathophysiology of vasogenic edema. AJNR Am J Neuroradiol 2008;29(6):1043-9.

[4] Bartynski WS, Boardman JF. Catheter angiography. MR angiography, and MR perfusion in posterior reversible encephalopathy syndrome. AJNR Am J Neuroradiol 2008;29(3):447-55.

[5] Mak A, Chan BP, Yeh IB, Ho RCM, Boey ML, Feng PH, et al. Neuropsychiatric lupus and reversible posterior leucoencephalopathy syndrome: a challenging clinical dilemma. Rheumatology (Oxford) 2008;47(3):256-62.

[6] Zhang YX, Liu JR, Ding MP, Huang J, Zhang M, Jansen O, et al. Reversible posterior encephalopathy syndrome in systemic lupus erythematosus and lupus nephritis. Intern Med 2008; 47(9):867-75.

[7] Bousser MG, Ferro JM. Cerebral venous thrombosis: an update. Lancet Neurol 2007;6(2):162-70.

[8] Leach JL, Fortuna RB, Jones BV, Gaskill-Shipley MF. Imaging of cerebral venous thrombosis: current techniques, spectrum of findings, and diagnostic pitfalls. Radiographics 2006;26 (Suppl 1):S19-41.

[9] Nieman LK, Biller BM, Findling JW, Newell-Price J, Savage MO, Stewart PM, et al. The diagnosis of Cushing's syndrome: an Endocrine Society Clinical Practice Guideline. J Clin Endocrinol Metab 2008;93(5):1526-40.

[10] Sundström T, Guez M, Hildingsson C, Toolanen G, Nyberg L, Riklund K. Altered cerebral blood flow in chronic neck pain patients but not in whiplash patients: a 99mTc-HMPAO rCBF study. Eur Spine J 2006;15(8):1189-95.

[11] Gooren LJ, Giltay EJ, Bunck MC. Long-term treatment of transsexuals with cross-sex hormones: extensive personal experience. J Clin Endocrinol Metab 2008;93(1):19-25. 